UCRL-CR-128684

S/C- B331058

\title{
Ultrashort X-Ray Backlighters and Applications
}

\author{
Donald Umstadter \\ University of Michigan
}

Submitted as Final Report on the DOE/LLNL Contract W-7405-ENG-48, Subcontract No. B331058 for period covering 8/1/96 - 7/31/97 


\section{DISCLAIMER}

This document was prepared as an account of work sponsored by an agency of the United States Government. Neither the United States Government nor the University of California nor any of their employees, makes any warranty, express or implied, or assumes any legal liability or responsibility for the accuracy, completeness, or usefulness of any information, apparatus, product, or process disclosed, or represents that its use would not infringe privately owned rights. Reference herein to any specific commercial product, process, or service by trade name, trademark, manufacturer, or otherwise, does not necessarily constitute or imply its endorsement, recommendation, or favoring by the United States Government or the University of California. The views and opinions of authors expressed herein do not necessarily state or reflect those of the United States Government or the University of California, and shall not be used for advertising or product endorsement purposes.

Work performed under the auspices of the U.S. Department of Energy by Lawrence Livermore National Laboratory under Contract W-7405-ENG-48. 


\title{
Ultrashort X-Ray Backlighters and Applications
}

Final Report on the DOE/LLNL Contract W-7405-ENG-48, Subcontract No. B331058

Covering the Period 8/1/96-7/31/97

\author{
Attn: Mr. Edwin Cassidy \\ Subcontract Administrator \\ Lawrence Livermore National Laboratory \\ P.O. Box 808, Mail Code L-650 \\ 7000 East Ave. \\ Livermore, CA 94551
}

Donald Umstadter, P.I.

Assoc. Professor

Center for Ultrafast Optical Science

1006 IST Bldg.

University of Michigan

Ann Arbor, MI 48109-2099

August 14, 1997 


\begin{abstract}
Previously, using ultrashort laser pulses focused onto solid targets, we have experimentally studied a controllable ultrafast broadband radiation source in the extreme ultraviolet for time-resolved dynamical studies in ultrafast science [J. Workman, A. Maksimchuk, X. Liu, U. Ellenberger, J. S. Coe, C.-Y. Chien, and D. Umstadter, "Control of Bright Picosecond X-Ray Emission from Intense Sub-Picosecond Laser-Plasma Interactions," Phys. Rev. Lett. 75, 2324 (1995)]. Once armed with a bright ultrafast broadband continuum $x$-ray source and appropriate detectors, we used the source as a backlighter to study a remotely produced plasma. The application of the source to a problem relevant to high-density matter completes the triad: creating and controlling, efficiently detecting, and applying the source. This work represented the first use of an ultrafast laser-produced $x$-ray source as a time- resolving probe in an application relevant to atomic, plasma and high-energy-density matter physics. Using the $\mathrm{x}$-ray source as a backlighter, we adopted a pump-probe geometry to investigate the dynamic changes in electronic stucture of a thin metallic film as it is perturbed by an ultrashort laser pulse. Because the laser deposits its energy in a skin depth of about $100 \AA$ before expansion occurs, up to gigabar pressure shock waves lasting picoseconds in duration have been predicted to form in these novel plasmas. This raises the possibility of studying high-energy-density matter relevant to inertial confinement fusion (ICF) and astrophysics in small-scale laboratory experiments. In the past, time-resolved measurements of K-edge shifts in plasmas driven by nanosecond pulses have been used to infer conditions in highly compressed materials. In this study, we used 100-fs laser pulses to impulsively drive shocks into a sample (an untamped $1000 \AA$ aluminum film on $2000 \AA$ of parylene-n), measuring L-edge shifts.
\end{abstract}




\section{Introduction}

We demonstrate the first use of a laser-produced $x$-ray source as a timeresolving probe in an application relevant to atomic, plasma and high-energydensity matter physics. Using the $\mathrm{x}$-ray source as a backlighter, we adopt a pump-probe geometry to investigate the dynamic changes in electronic structure of a thin metallic film as it is perturbed by an ultrashort-laser pulse. Because the laser deposits its energy in a skin depth of about $100 \AA$ before expansion occurs, very large amplitude shock waves can be generated without material pre-heat, commonly found in long pulse interactions [1]. Under these novel conditions, predictions indicate the generation of up to gigabar pressure shock waves lasting picoseconds in duration $[2,3]$. This raises the possibility of studying high energy density matter, relevant to inertial confinement fusion (ICF) as well as astrophysical states of matter in small-scale laboratory experiments with picosecond temporal resolution.

\section{Laser Compression and Shock Generation}

Plasma densities above solid, and electron temperatures below the Fermi temperature (defined below) can occur when the ablative force from a laserheated solid generates a shock wave traveling in the opposite direction, into the solid. The process of shock generation can be thought of as a wave induced by a moving piston. The force and velocity of the piston will be determined by the velocity and density of material ablating into the vacuum; this is Newton's third law, or the "rocket effect". The pressure due to thermal expansion (ablation), $P_{t h}$, can be determined from the ideal gas equation-of-state $P_{t h}=n_{e} k T_{e}$. For an ultrashort-pulse laser interaction with a solid, the density at which the laser is absorbed can be close to solid. With temperatures in the range of about $1000 \mathrm{eV}$ and a density of $10^{22} . \mathrm{cm}^{-3}$, one obtains a pressure of $10^{13}$ dynes $/ \mathrm{cm}^{2}$ or approximately ten-million atmospheres. There are few conventional means of obtaining pressures this high without thermonuclear detonations.

\section{Experimental Techniques}

Experiments for measuring hot dense matter, and, in particular, shock wave properties, include both optical and x-ray diagnostics. Measurements of the rear-surface luminous emission $[4,5,6,7]$, reflectivity [8] and phase shifts[9] al- 
(a)

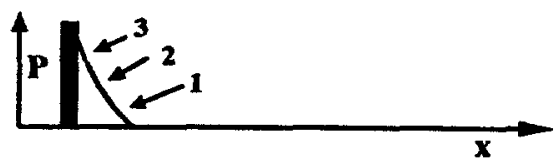

(b)

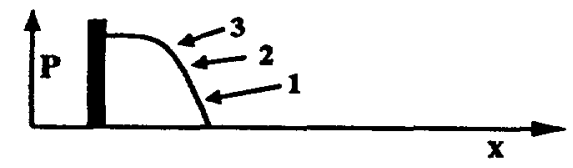

(c)

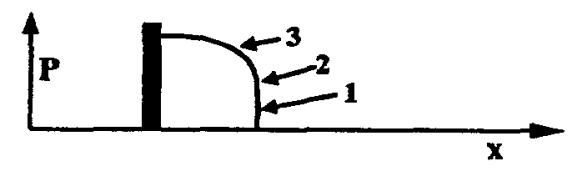

(d)

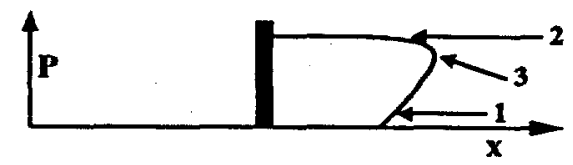

(f)

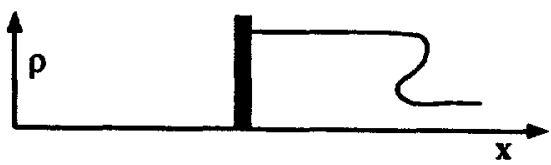

(e) $\uparrow \mathbf{P}$

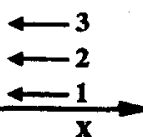

(g) $\uparrow \rho$

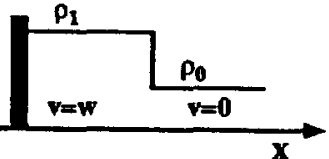

Figure 1: Gradual steepening of the pressure and density profile in a compression wa ve generated by an accelerating piston. Increasing sound speeds at points 1, 2 and 3 lead to "overshooting" as shown in (d), corresponding to a physical ly meaningless solution; (e) shows the actual profile with a discontinuity. (f) and (g) are the corresponding density profiles from (d) and (e). (Adapted from Zel'dovich and Raizer.)

low one to determine shock speed. Using thin targets of varying thicknesses, one can measure the time at which a shock arrives at the rear surface. This can be compared to the time at which the laser pulse arrives at the front surface to map out the evolving and steady-state shock speed. Direct measurements of shock speed and density can be obtained using x-ray[10, 11, 12] and optical[13] streak shadowgraphy. Using targets that are transparent to the backlighter under normal conditions (e.g. plastics or fused silica) one can determine density and its evolution, from changes in the opacity due to material compression and heating. Density measurements using EXAFS (extended $x$-ray absorption fine structure) $[14,15,16]$ rely on the spacing between scattering centers to show interference patterns in the absorbed $x$-ray spectrum. Shifts in the energy of photoabsorption edges[17, 18] can occur in dense materials due to a phenomenon known as continuum lowering. The 
dynamics of the edge shift can be used to infer properties of the shocked region.

The techniques mentioned have shown compression factors approaching three in solid targets with pressures as high as tens-of-megabars. It should be emphasized that all of the methods and measurements described here have been carried out with nanosecond laser interactions (except Ref. [9]) with intensities between $10^{13}-10^{15} \mathrm{~W} / \mathrm{cm}^{2}$. In the next section we present time-resolved experimental measurements of high-density matter using photoabsorption-edge shifts. The compression of an aluminum foil is accomplished using a $100-\mathrm{fs}$ laser pulse to generate a shock wave.

\section{Ultrashort Soft X-ray Backlighter in a Pump-Probe Ge- ometry}

In this section an experimental setup is described which was used for timeresolving a shift in the $L_{I I, I I I}$ photoabsorption edge in a thin aluminum foil, compressed by a 100 -fs laser-matter interaction. Aluminum is chosen as the target, due to its well-studied physical properties, its versatility and low-cost target construction. The $L$-edge was chosen instead of the $K$-edge for its accessibility to the soft $x$-ray source. The geometry uses a 100 fs laser pump and a time-resolving $x$-ray probe. This arrangement lends itself to almost any type of pump-probe $\mathrm{x}$-ray absorption measurements.

\section{Experimental Chamber}

As shown in Fig. 2, $50 \mathrm{~mJ}$ of the $100-\mathrm{fs} 10-\mathrm{Hz}$ Ti:Sapphire laser pulses were sent to a beamsplitter, where 90 percent of the energy was reflected onto an f/5 off-axis parabola, used to focus the light onto a solid gold target. The broadband ultrashort-pulse $x$-ray point source generated from this interaction was refocused to a sample at the opposite end of the experimental chamber using a section of a grazing incidence ellipsoidal optic; a central beam block eliminated any perturbations to the sample that might arise from hot electrons or hard $x$-rays produced by the laser-gold interaction. The remaining $5 \mathrm{~mJ}$ of laser light was sent into an optical delay line and then focused with an $\mathrm{f} / 10$ lens onto the thin metallic sample to an intensity of $1 \times 10^{14} \mathrm{~W} / \mathrm{cm}^{2}$. The sample plane was coincident with the slit of a variable spaced-grating grazing incidence imaging soft-x-ray spectrometer. The MCP coupled to 


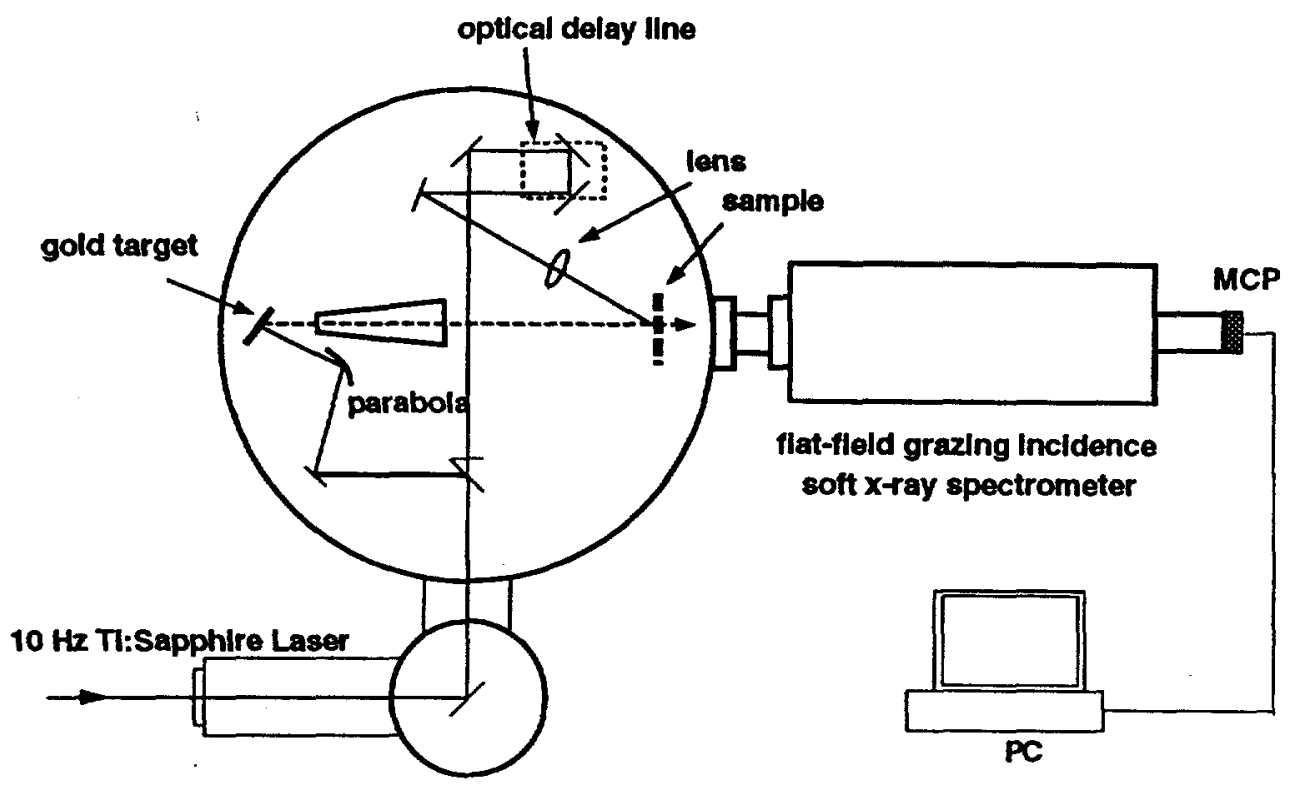

Figure 2: Experimental pump-probe setup for absorption spectroscopy (details in text).

the end of the soft $x$-ray spectrometer enabled us to record spectrally and spatially resolved $x$-ray absorption through the perturbed sample material. Time resolution was obtained by changing the arrival time of the laser pulse with respect to the $x$-ray pulse. As mentioned earlier, this experimental arrangement allows any type of sample to be probed, whether it be a gas jet, liquid, wire or thin film. In this experiment the sample consisted of 1000 $\AA$ of aluminum deposited onto $2000 \AA$ of parylene-n $\left(\mathrm{C}-\mathrm{H}_{n}\right)$, as a rear-side supporting structure. Due to the spatial resolution afforded by the spectrometer $(100 \mu \mathrm{m})$, comparison of absorption spectra through cold aluminum to that of the perturbed aluminum was possible for each data point. The laser focus of 125 microns, which was smaller than the 300-400 micron x-ray probe focus, allowed clear resolution of the perturbed region.

\section{X-ray Backlighter}

Broadband emission has previously been measured from 20 to $200 \AA[19,20]$, however, here we focus on the emission in the range of 100 to $200 \AA$, in the region of the aluminum $L_{I I, I I I}$-edge. The pulse duration was limited to 20 -ps. 
These longer pulse durations are due to intensity contrast limitations of the 790-nm laser light. Frequency doubling was found to be prohibitively inefficient (15 - 20 percent), possibly due to a combination of the short laser-pulse duration and KDP doubling crystal thickness. laser These measurements

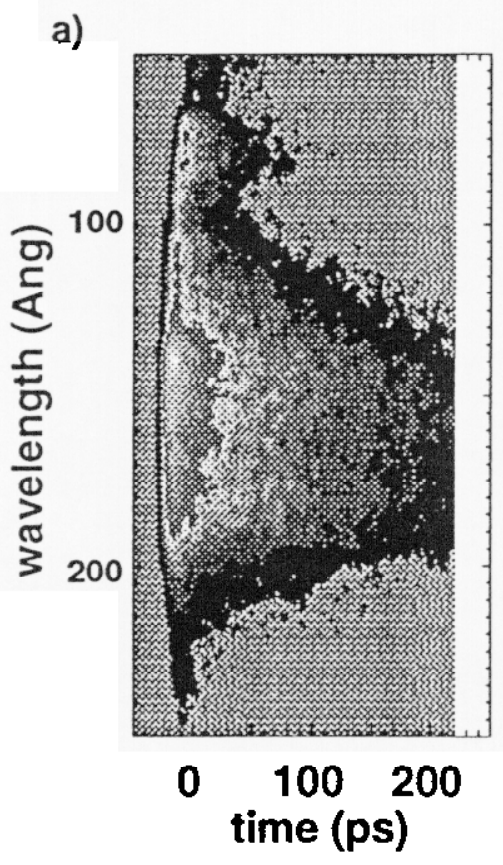

b)

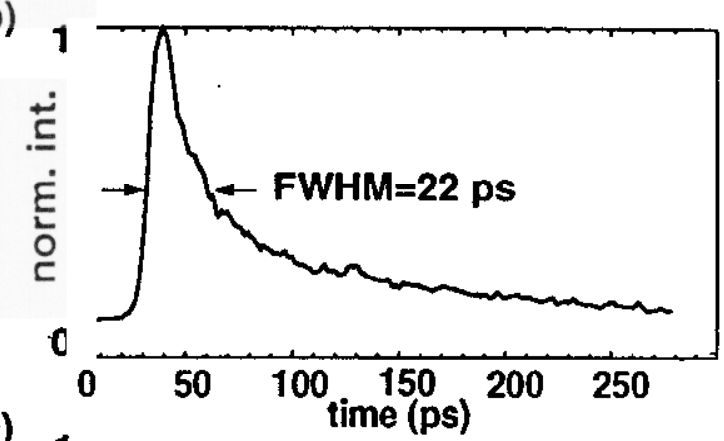

c)

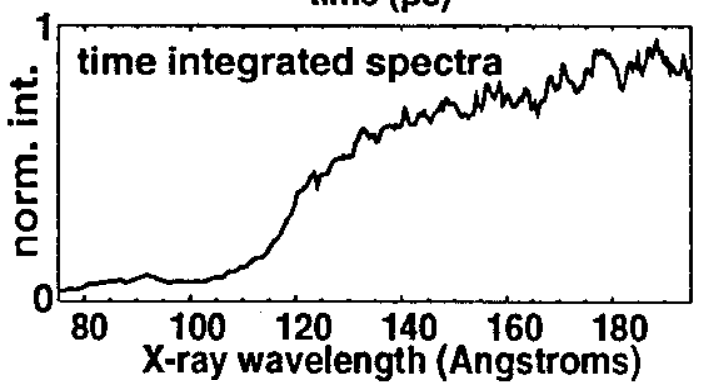

Figure 3: Soft x-ray source generated by a 100-fs laser interaction with a solid gold target. (a) Temporally and spectrally resolved images of the source. (b) Temporal lineout near $170 \AA$. (c) The spectrum was measured separately with a micro-channel-plate.

were obtained with the $x$-ray jitter-free streak camera[21]. The image shown in Fig. 3 is an accumulation of 20 interactions. In Fig. 3(a), the curvature due to different electron paths in the streak camera has not been corrected. The time-integrated spectrum in Fig.3(c) was obtained with the micro-channelplate (MCP) used for the pump-probe measurements. The decrease in spectral intensity in Fig.3(a) and (c) is due to the spectral intensity response of the spectrometer grating and detectors. 


\section{Experimental Observation of Photoabsorption-Edge Shifts in Compressed Aluminum}

The absorption feature used for analysis of the aluminum sample was the $\mathrm{L}_{I I, I I I}$ photoabsorption-edge at $72.78 \mathrm{eV}(170.04 \AA)[22]$. The position of the edge gives a direct measure of the ionization potential, and, thus, the electronic structure of the probed sample. The energy of the edge will change as

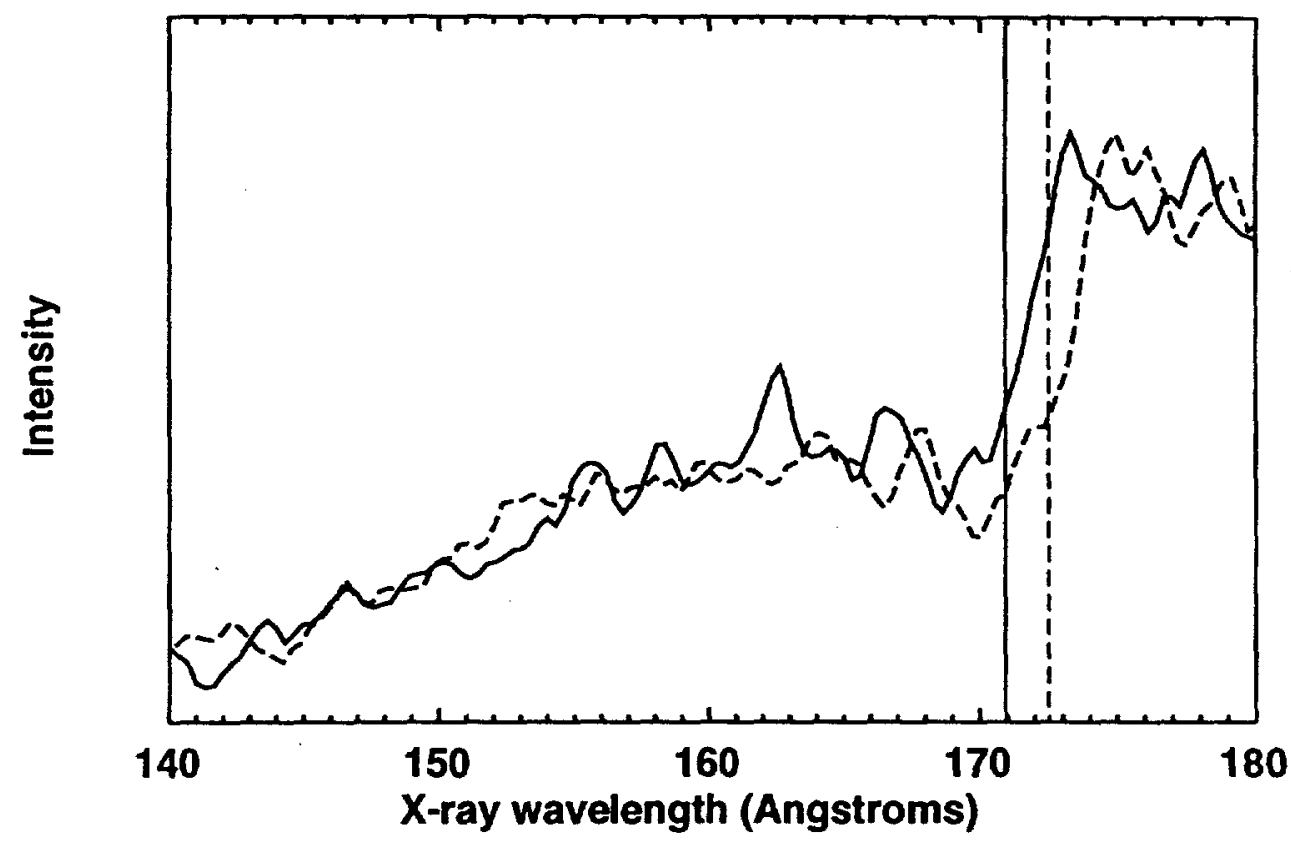

Figure 4: Comparison of the transmitted x-ray backlighter spectrum showing the maximum red shift(dashed line) and the cold aluminum edge position (solid line).

a function of material density, temperature and ionization state[17, 18]. Figure 4 shows the $x$-ray backlighter spectrum, generated from the gold interaction, after transmission through the thin aluminum sample. The two spectra correspond to transmission before arrival of the laser pulse (solid line) and transmission just after the laser pulse has compressed the aluminum (dashed line). The laser-pump intensity used was $1 \times 10^{14} \mathrm{~W} / \mathrm{cm}^{2}$, consistent with previous longer-pulse work. After the laser pulse has arrived, the spectral position of the photoabsorption edge has shifted to longer wavelength by $1.6 \AA$ (red shifted). The measured spectral shift of the $L_{I I, I I I}$-edge, with respect 


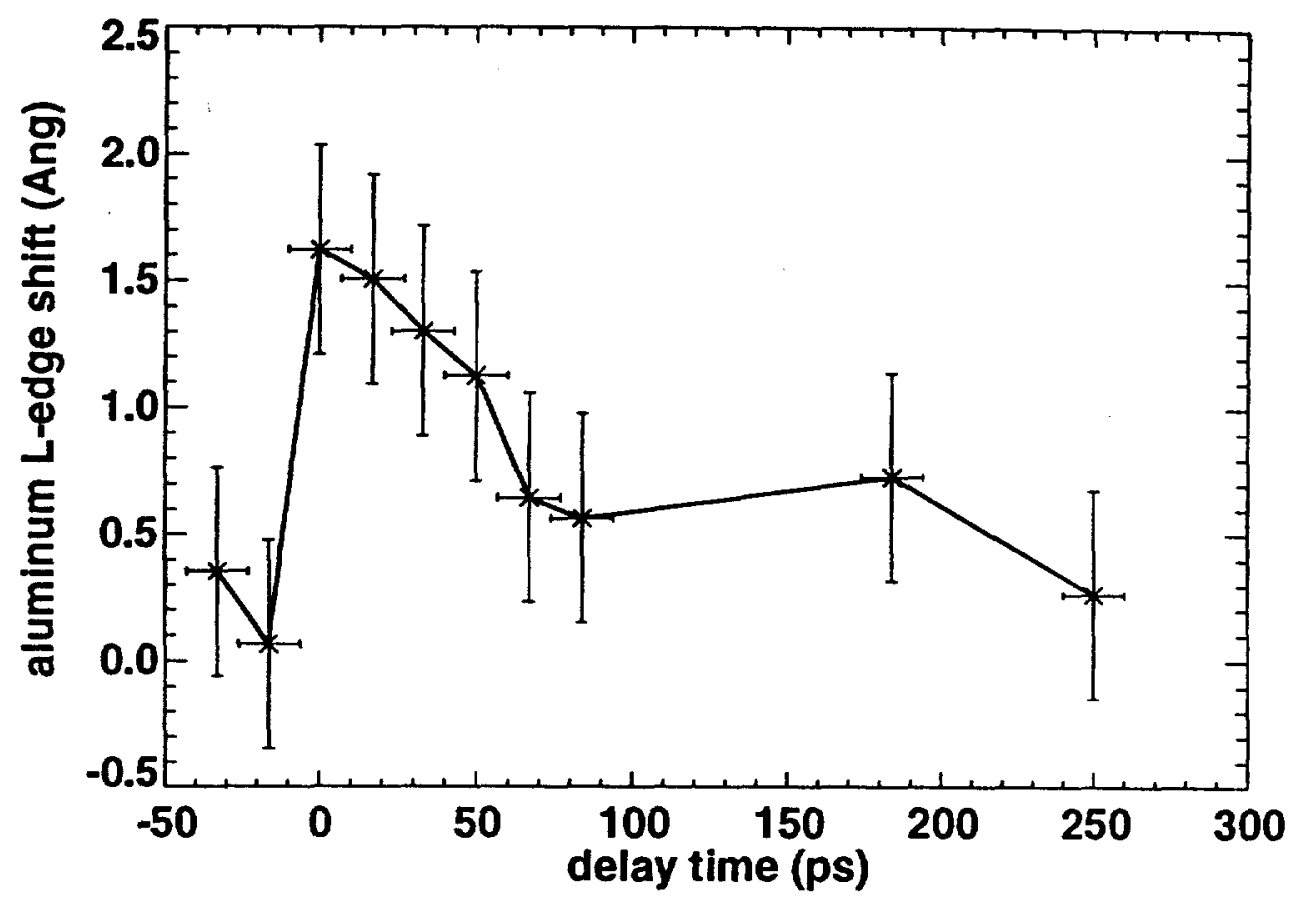

Figure 5: Red shift of the aluminum $\mathrm{L}_{I I, I I I}$ edge as a function of $\mathrm{x}$-ray probe delay time.

to its cold, neutral position, is recorded as a function of probe delay in Fig.5. Each data point consisted of 10 to 20 laser shots. Error bars indicate the time resolution of the 20-ps x-ray probe on the time axis and the averaging of several data files on the shift axis. The early times $(t<0)$ correspond to probing unperturbed sample with the soft $\mathrm{x}$-ray source before the laser arrives. We then see a maximum red-shift of $1.6 \pm 0.4 \AA$, which decreases at later times as the sample decompresses. The shift of $1.6 \AA$ is much greater than one would expect due to the Doppler shift of an expanding material. For expansion velocities as high as $10^{6} \mathrm{~cm} / \mathrm{sec}$ (one might expect at this intensity) a maximum Doppler shift at $170 \AA$ would correspond to $0.017 \AA$. The spectral position of the photoabsorption edge falls back to its cold position within approximately 50 -ps. This time-scale turns out to be consistent with propagation of a disturbance through a cold $1000 \AA$ aluminum sample at the sound speed. 


\section{Contributions to the Photoabsorption Edge Shift}

To gain an understanding of the experimental results, we use an analytic plasma model to describe the relationship between L-edge position and the associated plasma parameters; temperature, density and ionization. Because the hydrodynamics code does not include a reasonable equation-of-state for high densities and low temperatures, nor can it handle shock waves[23], the following analysis will rely primarily on the analytical model. Reference to hydrodynamics simulations from a better adapted code (CHIVAS), corroborates our understanding of the shock and relaxation dynamics. Our analytical analysis depends on three basic phenomena: Ionization, which generates blue spectral shifts, continuum lowering, which contributes red shifting and electron degeneracy, which takes into account some solid-state effects. The total shift in the ionization potential, and, thus, the photoabsorption edge, will be given by the sum of these three contributions[17]

$$
\Delta E_{\text {tot }}=\Delta E_{\text {ion }}+\Delta E_{c l}+\Delta E_{\text {deg }},
$$

where $\Delta E_{i o n}$ is the shift due to ionization, $\Delta E_{c l}$ is the shift due to continuum lowering and $\Delta E_{\text {deg }}$ is the shift due to electron degeneracy.

The three contributions described in the previous section sum together to give a total shift, $\Delta E_{\text {tot }}=\Delta E_{\text {ion }}+\Delta E_{\text {cl }}+\Delta E_{\text {deg }}[17]$, as stated above. The total shift is, therefore, a function of electron temperature, density and ionization state, giving a three dimensional parameter space which changes with time. Taking the analytic model described in the previous section, one can plot the predicted shift as a function of density for a particular temperature and ionization state. Using the above plasma model, with Saha-based scaling for ionization as a function of temperature, we can plot the locus of points representing the maximum observed edge-shift as a function of temperature and ion density. The experimental error in the shift measurement is included in the width of the curve, determined by the model at a shift of $1.6 \pm 0.4$ $\AA$. This plot demonstrates the need for a measurement of the temperature to accurately determine the density, and hence the compression in our sample. Without such a complimentary measurement, detailed hydrodynamic information is necessary to isolate a single set of parameters describing the plasma. With the use of the detailed 1.5-D hydro code CHIVAS, we find a temperature and maximum compression which fall close to our experimental boundaries[24]. The discrepency between the analytic analysis and the numerical results may be due to the omission of band structure in the 
analytic model. The numerical results show consistency with our analytic approach and indicate a maximum compression of 1.4 times solid density at a temperature of $3.9 \mathrm{eV}$.

Of course, in our geometry an absorption measurement integrates the contributions from each region of the sample, which can be roughly divided into a high temperature low density blow-off region, a high density low temperature compressed region and the undisturbed cold material. High temperature regions contribute ionization blue shifts that are masked by red shifts. We therefore probe the higher density regions at any given time. In fact, we expect the measured shift to be a weighted average, resulting from contributions from different positions in the high density region.

Hydrodynamic calculations of 100 -fs laser generated shock waves indicate that the shock will be generated in picoseconds and will traverse the 1000 $\AA$ material on a time-scale of the order 5 to 10 picoseconds $[2,24,25]$. This gives a propagation speed of about $10^{6} \mathrm{~cm} / \mathrm{s}$, which in the cold material would correspond to a Mach number of about 2. This implies that our time-resolution is ideal for measuring the dynamics of the relaxation of the material after the shock, but not yet sufficient to resolve the dynamics of the shock wave itself.

\section{Nontechnical Aspects of the Report}

The work was done at the University of Michigan Center for Ultrafast Optical Science (CUOS), where a graduate student was supported by this contract. The following publication acknowledged the support of the contract, J. Workman, M. Nantel, A. Maksimchuk and D. Umstadter, "Application of a Picosecond X-Ray Source to Time-Resolved Plasma Dynamics," Appl. Phys. Lett. 70, 312 (1997). The results were also discussed in the following invited talk: "Atomic Processes in Ultrashort-Pulse Laser-Plasmas," International Conference on Laser Interaction and Related Plasma Phenomena, Monterey, CA, April, 1997.

\section{References}

[1] Ya. B. Zel'dovich and Yu. P. Raizer, Physics of Shock Waves and HighTemperature Hydrodynamic Phenomena (Academic Press, New York, 1967). 
[2] V. E. Gusev, Phys. Vibr. 57, 1 (1993).

[3] A. Ng, A. Forsman, and P. Celliers, Phys. Rev. E 51, R5208 (1995).

[4] A. Ng, D. Parfeniuk and L. DaSilva, Phys. Rev. Lett. 54, 2604 (1985).

[5] R. J. Trainor, J. W. Shaner, J. M. Auerbach and N. C. Holmes, Phys. Rev. Lett. 42, 1154 (1979).

[6] B. Arad, A. Borowitz, S. Eliezer, Y. Gazit, I. Gilath, M. Givon, S. Jacke 1, A. D. Krumbein and H. Szichman, Plasma Phys. Cont. Fusion 26, 845 (1984).

[7] F. Cottet, J. P. Romain, R. Fabbro and B. Faral, Phys. Rev. Lett. 52, 1884 (1984).

[8] M. Werdinger, B. Arad, E. Moshe and S. Eliezer, Quant. Electron. 25, 153 (1995).

[9] R. Evans, A. D. Badger, F. Falliès, M. Mahdieh, T. A. Hall, P. Audebert, J.-P. Geinder, J.-C. Gauthier, A. Mysyrowicz, G. Grillon and A. Antonetti, Phys. Rev. Lett. 77, 3359 (1996).

[10] R. Kodama, K. A. Tanaka, M. Nakai, K. Nishihara, T. Norimatsu, T. Yamanak a and S. Nakai, Phys. Fluids B 3, 735 (1991).

[11] B. A. Hammel, D. Griswold, O. L. Landen, T. S. Perry, B. A. Remington, P. L. Miller, T. A. Peyser and J. D. Kilkenny, Phys. Fluids B 5, 2259 (1993); B. A. Hammel, J. D. Kilkenny, D. Munro, B. A. Remington, H. N. Kornblum, T. S. Perry, D. W. Phillion and R. J. Wallace, Phys. Plasmas 1, 1662 (1994).

[12] J. Edwards, M. Dunne, R. Taylor, O. Willi, C. A. Back and S. J. Rose, Phys. Rev. Lett. 71, 3477 (1983).

[13] A. Ng, P. Celliers and D. Parfeniuk, Phys. Rev. Lett. 58, 214 (1987) .

[14] T. A. Hall, A. Djaoui, R. W. Eason, C. L. Jackson, B. Shiwai, S. L. Rose, A. Cole and P. Apte, Phys. Rev. Lett. 60, 2034 (1988). 
[15] A. Djaoui, B. Shiwai, T. A. Hall, R. W. Eason, C. Jackson and S. J. Rose, Plasma Phys. Cont. Fusion 31, 111 (1989); A. Djaoui, T. A. Hall. R. C. Albers, J. J. Rehr and J. Mustre, Laser Part. Beams 8, 319 (1990).

[16] F. I. Gordon, Plasma Phys. Cont. Fusion 35, 1207 (1993).

[17] D. K. Bradley, J. Kilkenny, S. Rose, and J. D. Hares, Phys. Rev. Lett. 59, 2995 (1987).

[18] L. DaSilva, A. Ng, B. K. Godwal, G. Chiu, and F. Cottet, M. C. Richardson, P. A. Jaanimagi and Y. T. Lee, Phys. Rev. Lett. 62, 1623 (1989).

[19] M. M. Murnane, H. C. Kapteyn, R. W. Falcone, Phys. Rev. Lett. 62, 155 (1989).

[20] J. Workman, A. Maksimchuk, X. Liu, U. Ellenberger, J. S. Coe, C. Y. Chien, and D. Umstadter, Phys. Rev. Lett. 75, 2324 (1995).

[21] A. Maksimchuk, M. Kim, J. Workman, G. Korn, J. Squire, D. Du, D. Umstadter, G. Mourou, and M. Bouvier, Rev. Sci. Instrum. 67, 697 (1996).

[22] B. L. Henke, P. Lee, T. J. Tanaka, R. L. Shimabukuro, and B. K. Fujikawa, J. Opt. Soc. Am. B 3, No. 11, 1540 (1986).

[23] The hydrodynamics code described uses artificial viscocity to damp out shock waves. This is done to avoid numerical error from steep gradients.

[24] S. Jacquemot and A. Decoster, private communication, 1996.

[25] J. C. Gauthier, private communication, 1996.

[26] These are also known as Kronig and Kossel structure, respectively. 


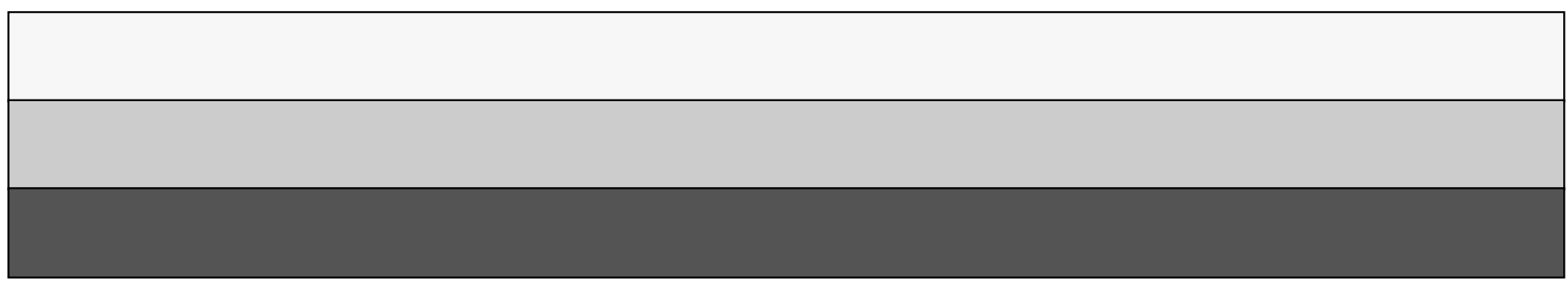

